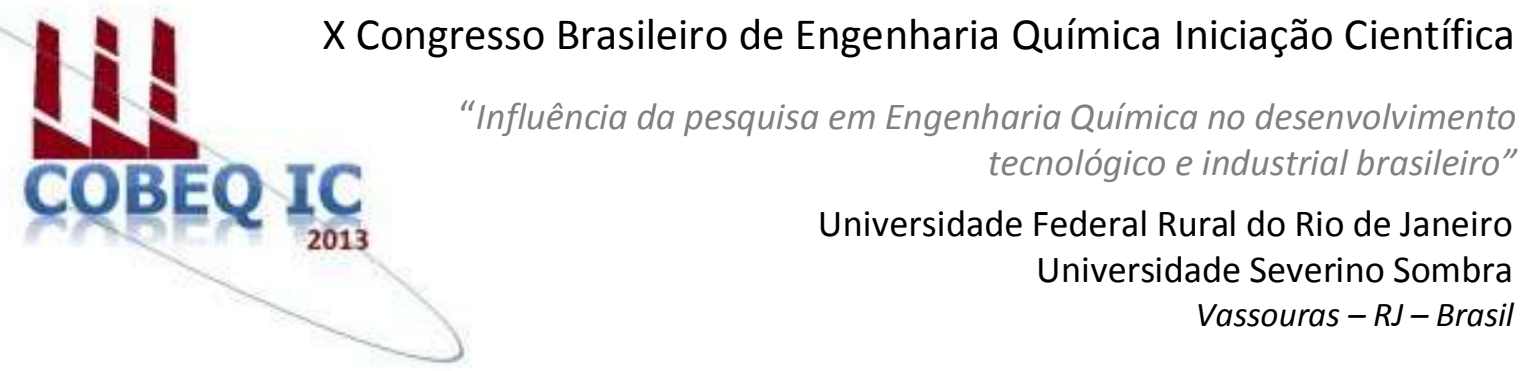

\title{
PLANEJAMENTO EXPERIMENTAL DO PROCESSO DE ELETROCOAGULAÇÃO NO TRATAMENTO DE CHORUME DE ATERRO SANITÁRIO. ${ }^{(*)}$
}

\author{
PORTO $^{1}$, T. M.; YASSUE ${ }^{1}$, P. H.; THEODORO ${ }^{2}$, P. S.; \\ MODENES ${ }^{3}$, A. N.; BRAGIÃO ${ }^{1}$, M. E.
}

\begin{abstract}
(1) Acadêmica Engenharia Química - UNIOESTE; ${ }^{(2)}$ Engenheiro Químico; ${ }^{(3)}$ Docente DEQ/UNIOESTE Departamento de Engenharia Química - Universidade Estadual do Oeste do Paraná - UNIOESTE, Rua da Faculdade,645 - CEP 85903000 - e-mail: anmodenes@yahoo.com.br
\end{abstract}

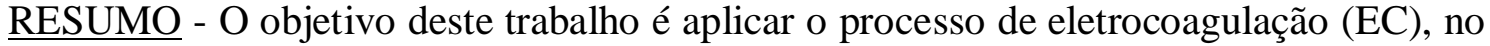
tratamento de chorume de aterro sanitário. Este é um efluente de alta carga orgânica e que provoca alguns males ambientais, e com isso, processos de tratamento sempre estão sendo investigados. A coleta do efluente bruto foi realizada no aterro sanitário da cidade de Cascavel - Pr. O processo foi realizado em escala laboratorial e para avaliar as melhores condições de processo do sistema, foi aplicado um planejamento experimental $3^{3}$ completo. Para realização dos experimentos EC, em batelada, foi utilizado um reator de material plástico com uma capacidade de 2 litros de efluente para ser tratado, provido de uma torneira para fácil escoamento do lodo gerado após o tratamento de EC. Os eletrodos, tipo colmeia, foram confeccionados com placas de ferro, com uma largura de $5 \mathrm{~cm}$, altura de 15 e espessura de $3 \mathrm{~mm}$. A distância entre as 6 placas de ferro foi de 2,5 $\mathrm{cm}$. A eficiência do processo foi avaliada com base na redução de demanda química de oxigênio (DQO), cor e turbidez. Os parâmetros operacionais avaliados no reator de eletrocoagulação foram: o $\mathrm{pH}$ inicial, tempo e intensidade de corrente. O processo mostra-se eficiente para o tratamento de chorume, devido a facilidade e baixo custo de operação. A maior eficiência na redução dos poluentes verificada no processo de eletrocoagulação foi de 75, 82 e $60 \%$ para turbidez, cor e DQO respectivamente, sendo remoções satisfatórias para o tratamento.
\end{abstract}

\section{Palavras chave: EFICIÊNCIA, REMOÇÃO, PARÂMETROS.}

\section{INTRODUÇÃO}

A produção de resíduos está cada vez mais acentuada, e com isso a eliminação deles em aterro sanitário é uma parte importante e essencial em todo mundo.

Porém, um dos mais graves problemas ambientais relacionados ao aterro é a geração de lixiviados durante o processo de decomposição pela água da chuva que percolam, atingindo as camadas do solo.

O chorume é um líquido escuro, de odor desagradável, contendo alta carga orgânica e inorgânica. A composição química e microbiológica do chorume é bastante 
complexa e variável, uma vez que, além de depender das características dos resíduos depositados, é influenciada pelas condições ambientais, pela forma de operação e idade do aterro e, principalmente, pela dinâmica dos processos de decomposição que ocorrem no interior das células (EL FADEL et al., 2002; KJELDSEN et al., 2002; TATSI et AL., 2003).

Os processos convencionais utilizados no tratamento do chorume são baseados em processos físico-químicos e biológicos. Os primeiros costumam ser fundamentados em processos de adsorção e em rotinas de precipitação-floculação, que apresentam elevada eficiência de depuração. Entretanto, as substâncias contaminantes não são degradadas, o que necessariamente implica a geração de fases sólidas (lodos) altamente contaminados (BAE et al, 1999). Processos biológicos, por sua vez, podem ser aplicados na forma de rotinas aeróbias, anaeróbias e facultativas (FREIRE et al, 2000; STROOT et al, 2001). Infelizmente, características como necessidade de longos tempos de residência (variando de dias até semanas) e baixa eficiência na remoção de compostos recalcitrantes e coloridos (FREIRE et al, 2000), fazem com que a sua eficiência seja bastante discutida.

As técnicas tradicionais aplicadas no tratamento dos efluentes em geral, tais como coagulação/floculação, separação por membranas (ultra filtração, osmose inversa) ou a eliminação por carvão ativado (adsorção), apenas transferem o poluente de fase, e os tratamentos biológicos também não apresentam degradação satisfatória (GARCIA et al, 2008; KNITTEL E SCHOLLMEYER, 2008; NEELEVANNAN et al, 2007). Com isso, é constante a busca por novas tecnologias que equilibrem o custo e a eficiência, entre as quais se destaca a eletro-coagulação (EC), combinando a oxidação parcial do poluente, via eletrolítica, com a precipitação físicoquímica ou eletroquímica do lodo (ALINSAFI et. al., 2005).

A EC tem hoje uma ampla gama de aplicações, dentre as quais se destacam: efluentes têxteis (RAGHU et al., 2007, RAJU et al., 2008 ), águas residuais de lavanderias (GE et al., 2004), remoção de íon fluoreto (SHEN et al.,2003) e tratamentos de efluentes de curtumes (MURUGANANTHAN et al., 2004, ESPINOZA-QUIÑONES, et al., 2009).

\section{MATERIAIS E MÉTODOS}

A coleta do efluente foi efetuada no aterro sanitário da cidade de Cascavel, Pr, localizada na região oeste do estado. $\mathrm{O}$ aterro é constituído de várias lagoas, sendo elas, de chorume bruto, em tratamento e tratado. A mostra coletada foi de chorume bruto.

A caracterização físico-química do efluente foi realizada nos laboratórios de Controle de poluição (CP) e no Núcleo de Biotecnologia e Desenvolvimento de processos químicos (NBQ) da Universidade Estadual do Oeste do Paraná - Unioeste Campus de Toledo. Sendo os parâmetros analisados $\mathrm{pH}$, cor, turbidez e DQO. Também foram realizadas corridas para testes com tempos diversificados, para a partir disso elaborar um planejamento experimental.

Todas as análises físico-químicas foram realizadas de acordo com a metodologia descrita no Standard Methods. Foi realizado um planejamento experimental $3^{3}$ para a realização do estudo do processo de eletrocoagulação.

Para realização dos experimentos EC, em batelada, foi utilizado um reator de material plástico com uma capacidade de 2 litros de efluente para ser tratado, provido de uma torneira para fácil escoamento do lodo gerado após o tratamento de EC. Os eletrodos, tipo colmeia, foram confeccionados com placas de ferro, com uma largura de $5 \mathrm{~cm}$, altura de 15 e espessura de $3 \mathrm{~mm}$. A distância entre as 6 placas de ferro foi de $2,5 \mathrm{~cm}$.

\section{RESULTADOS E DISCUSSÕES}

Através da Tabela 1 é apresentado os resultados da caracterização do efluente Bruto, para os seguintes parâmetros: Cor, Turbidez, DQO.

Tabela 1- Resultados da caracterização do Efluente Bruto.

\begin{tabular}{c|c|c}
\hline B & Valor & Unidade \\
\hline Cor & 331 & Pt-Cor \\
Turbidez & 160 & NTU \\
DQO & 1420 & $\mathrm{mgL}^{-1}$ \\
\hline
\end{tabular}


A matriz experimental foi desenvolvida aplicando o planejamento fatorial $3^{3}$. O experimento consistiu na análise dos efeitos dos fatores corrente (i), $\mathrm{pH}$ e Tempo(min) sobre os parâmetros resposta, avaliados em três níveis apresentados pela Tabela 2 juntamente com os respectivos coeficientes.

Tabela 2 - Fatores e Níveis aplicados ao experimento.

\begin{tabular}{ccccc} 
& & \multicolumn{3}{c}{ Níveis } \\
\hline Fator & Coef & -1 & 0 & 1 \\
\hline Tempo $(\min )$ & F1 & 5 & 32,5 & 60 \\
Corrente $(\mathrm{A})$ & F2 & 0,5 & 2,75 & 5 \\
pH & F3 & 3 & 6 & 9 \\
\hline
\end{tabular}

O modelo matemático de regressão (função resposta) do referido planejamento é representado pela equação 1 .

$$
y=\beta_{0}+\sum_{i=1}^{k} \beta_{i} x_{i}+\sum_{i=1}^{k} \beta_{i j} x_{i}^{2}+\sum_{i=1}^{k-1} \sum_{j>1}^{k} \beta_{i j} x_{i} x_{j}+\varepsilon
$$

Os modelos de regressão obtidos por meio das análises estatísticas, fazem a correlação entre a função resposta (percentual de remoção da Cor, Turbidez e DQO) e os fatores significativos (pH, Tempo e Corrente elétrica) juntamente com as interações entre os mesmos. A validade dos modelos matemáticos gerados foi comprovado através da análise de variância (ANOVA). Para que o modelo seja considerado válido dentro do intervalo de confiança (Fcalculado) deve ser maior que o fator de (Ftabelado) ou o valor de $\mathrm{p}<0,05$, conforme apresentado na Tabela 3.

Tabela 3- ANOVA Análise de Variância do modelo de remoção da Turbidez,Cor e DQO

\begin{tabular}{|c|c|c|c|c|c|c|c|}
\hline & $\begin{array}{l}\text { Fonte de } \\
\text { Variação }\end{array}$ & $\begin{array}{c}\text { Soma } \\
\text { Quadrática }\end{array}$ & $\begin{array}{c}\text { Graus de } \\
\text { Liberdade }\end{array}$ & $\begin{array}{l}\text { Média } \\
\text { Quad. }\end{array}$ & $\begin{array}{c}\text { F } \\
\text { Calc. }\end{array}$ & $\begin{array}{c}\text { F } \\
\text { Tab }\end{array}$ & p-Valor \\
\hline \multirow{3}{*}{ Cor } & Regressão & 8054 & 4 & 2013,56 & 5,7973 & & $2,42.10^{-3}$ \\
\hline & Erro & 7641,16 & 22 & 347,326 & & - & \\
\hline & Total SS & 15695,41 & 26 & & & & \\
\hline \multirow{3}{*}{ Turbidez } & Regressão & 8070 & 4 & 2017,41 & 5,8201 & & $2,37.10^{-3}$ \\
\hline & Erro & 7625,78 & 22 & 346,626 & & - & \\
\hline & Total SS & 15695,41 & 26 & & & & \\
\hline \multirow{3}{*}{ DQO } & Regressão & 2060762 & 25 & 82430,4 & 5,9147 & & $2,07.10^{-8}$ \\
\hline & Erro & 766516 & 55 & 13936,6 & & - & \\
\hline & Total SS & 2827278 & 80 & & & & \\
\hline
\end{tabular}

As Figuras 1- 3 apresentam a superfície de resposta multiparamétrica da percentagem de remoção da DQO, cor e turbidez em função da variação dos seguintes parâmetros tempo de eletrólise, corrente (A) e pH, quando significativa $(p<0,05)$, e das interações entre esses parâmetros quando também significativas. Por meio destas figuras, é possível observar o comportamento dos processos de remoção.

\section{Turbidez}


Na Figura 1, é apresentada as variações das percentagens de remoção da turbidez. Conforme esta Figura observa-se que a máxima porcentagem de remoção foi de $75 \%$ de turbidez.

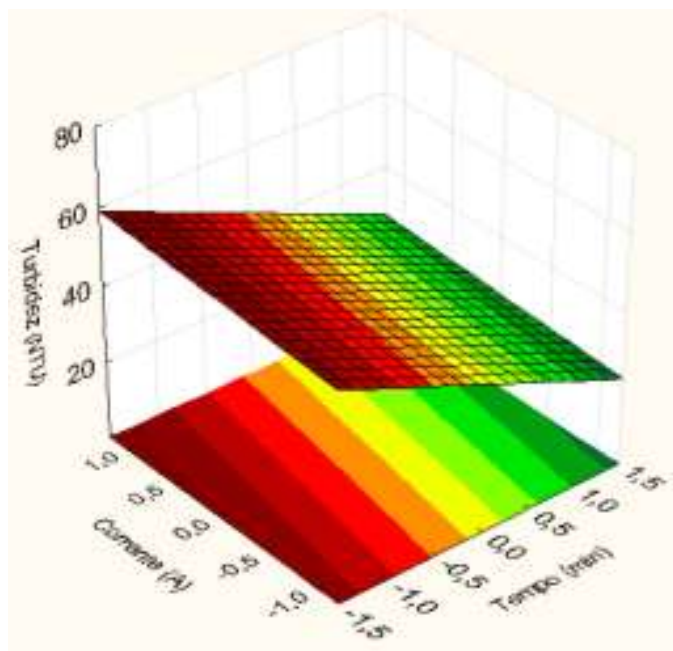

Figura 1 - Superfícies de resposta para remoção da turbidez.

\section{Cor}

$\mathrm{Na}$ Figura 2 foi mantido constante o $\mathrm{pH}$. A variação da percentagem de remoção da cor está sob a influência do tempo e sob a influência da corrente. Conforme esta Figura, observa-se que a máxima porcentagem de remoção esta numa região compreendida da corrente entre 2 e $4 \mathrm{~A}$.

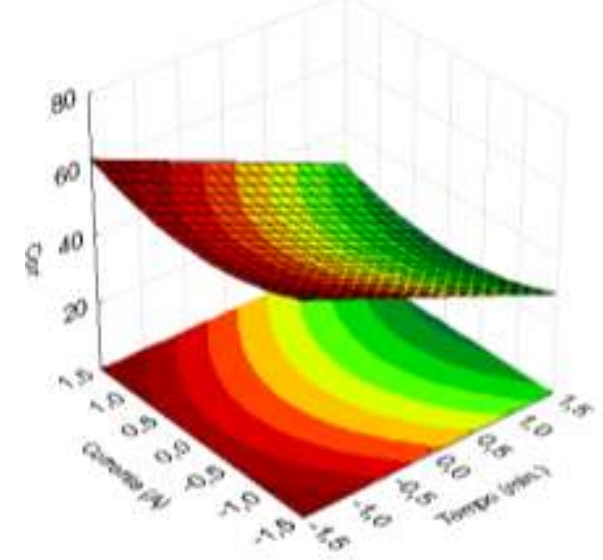

Figura 2-Superfícies de resposta para remoção da cor.

\section{DQO}

$\mathrm{Na}$ Figura 3 foi mantido constante o tempo. A variação da percentagem de remoção da DQO está sob a influência da corrente e sob a influência do $\mathrm{pH}$. Conforme esta Figura, observa-se que a máxima porcentagem de remoção esta numa região compreendida no valor de $\mathrm{pH}$ entre 6 e 9 e corrente entre 2 e $4 \mathrm{~A}$.

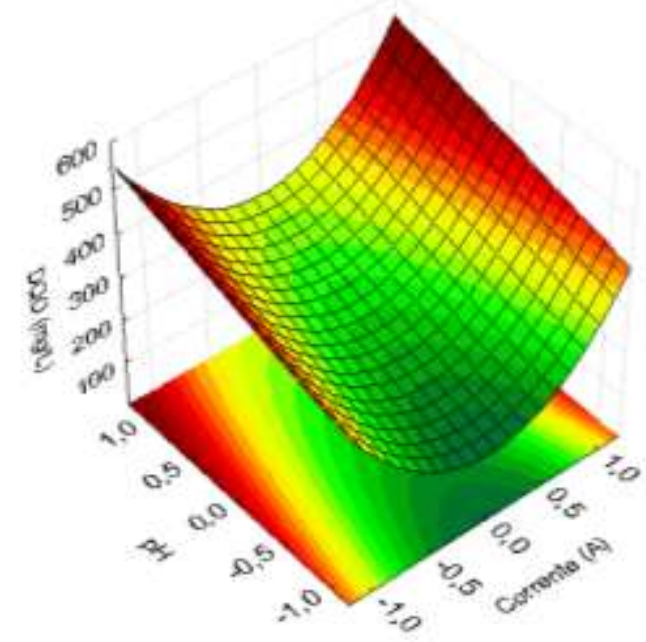

Figura 3-Superfícies de resposta para remoção da turbidez.

\section{CONCLUSÃO}

O planejamento experimental completo $3^{3}$ determinou as condições ótimas de processo: $\mathrm{pH}$ inicial 9,0 ; tempo de eletrólise de 5 minutos e intensidade de corrente de $5 \mathrm{~A}$, obtendo assim os melhores resultados na remoção da DQO, cor e turbidez, que são 60, 82 e $75 \%$, sendo assim: $568 \mathrm{mgL}^{-1}, 59,58 \mathrm{Pt}-$ Cor e 40 NTU respectivamente. A análise de viabilidade indicou que o tratamento de efluentes via processo de eletrocoagulação é mais eficiente quando se trabalha com maiores volumes de efluente a ser tratado justificando assim a viabilidade de se aplicar esta técnica em escala industrial.

Conclui-se que todos os objetivos traçados no planejamento do projeto foram alcançados satisfatoriamente, sendo que os resultados obtidos, de um modo geral, mostram que o tratamento utilizado pode ser considerado eficiente no tratamento de chorume de aterro sanitário.

\section{REFERERÊNCIAS}

ALINSAFI, A., KHEMIS, M., PONS, M. N., PONS, M. N., LECLERE, J. P., YAACOUBI, A., BENHAMMOU, A., 
NEJMEDDINE, A., Electrocoagulation of reactive of reactive textile dyes and textile wastewater, Chemical Engineering and Processing 44, 461-470 p., 2005.

BAE, B., JUNG, E. S., KIM, Y. R., SHIN, H. S. Treatment of Landfill Leachate Using Actived Sludge Process and ElectroBean Radiation. Water Research. Vol. 33, (11), p. 2669 - 2673, 1999.

EL FADEL, M.; DOUSEID, E.; CHAHINE, W.; ALAYLIC, B. Factors influencing solid waste generation and management. Waste Management. V. 22, p. 269 276, 2002.

ESPINOZA-QUIÑONES, F. R., FORNARI, M. M. T., MÓDENES, A. N., PALÁCIO, S. M., SILVA, F. G., SZYMANSKI, N., KROUMOV, A.D., TRIGUEIROS, D. E. G. Pollutant removal from tannery effluent by Electrocoagulation. Chemical Engineering Journal. 151. 59-65 p., 2009.

FREIRE, R. S. et al. P. Novas tendências para $o$ tratamento de resíduos industriais contendo espécies organocloradas. Química Nova. v. 23, n. 4, p. 504 - 511, 2000.

GARCIA, J. C., SIMIONATO, J. I., CARLI DA SILVA, A. E., NOZAKI, J., DE SOUZA, N. E., Solar photocatalytic degradation of real textile effluents by associated titanium dioxide and hydrogen peroxide. Solar Energy, article in press., 2008.

GE, J., Qu, J., Lei, P. and Liu, H., New bipolar electroflotation treatment of Laundry wastewater. Sep. Purif. Technol. 36 (1),33-39 p., 2004.

KJELDSEN, P.; BARLAZ, M. A.; ROOKER, A. P.; BAUN, A.; LEDIN, A.; CHRISTENSEN, T. Present and LongTerm Composition of MSW Landfill Leachate: A Review. Environmental Science and Technology. V. 32, (4), p. 297 - 336, 2002.

KNITTEL, D., and SCHOLLMEYER, E., Functional group analysis on oxidized surfaces of synthetic textile polymers.
Journal of Hazardous Materials, 154, 83-91 p., 2008.

MURUGANANTHAN, M., RAJU, G. B., and PRABHAKAR, S., Removal of sulfide, sulfate and sulfite ions by electro coagulation. Journal Hazard. Mater. 109 (1-3), 37-44., 2004.

NEELAVANNAN, M. G., REVATHI, M., BASHA, C.A., Photocatalytic and electrochemical combined treatment of textile wash water. Journal of Hazardous Materials, 149, 371-378 p., 2007.

RAGHU, S., BASHA, C. A., Chemical or electrochemical techniques, followed by ion exchange, for recycle of textile dye wastewater. Journal Hazardous Materials. 149, 324-330 p., 2007.

SHEN, F., GAO, P., CHEN, X., Removal of chromium (VI) from wastewater by combined electocoagulationeletroflotation without a filter. Sep. Purif. Technol. 43 (2), 117-123 p., 2005.

STROOT, P. G. et al. Anaerobic Condigestion of Municipal, Solid Waste and Biosolids Under Various Mixing Conditions - II. Microbial Population Dynamics. Water Research. v. 35, n. 7, p. $1817-1827$, 2001.

TATSI, A. A; Zouboulis, A. I.; Matis, K. A.; Samaras, P.; "Coagulationflocculation pretreatment of sanitary landfill leachates", Chemosphere 53 , 737-744, 2003.

\section{AGRADECIMENTOS}

Agradeço ao CNPQ pelo apoio financeiro. 